\title{
Optimization Study of Multidimensional Big Data Matrix Model in Enterprise Performance Evaluation System
}

\author{
Honglin Fu \\ Hong Kong Baptist University, Kowloon Tong, Kowloon, Hong Kong \\ Correspondence should be addressed to Honglin Fu; fuhonglin0903@163.com
}

Received 15 July 2021; Revised 1 August 2021; Accepted 10 August 2021; Published 27 August 2021

Academic Editor: Shan Zhong

Copyright ( 2021 Honglin Fu. This is an open access article distributed under the Creative Commons Attribution License, which permits unrestricted use, distribution, and reproduction in any medium, provided the original work is properly cited.

\begin{abstract}
This paper uses a multidimensional big data matrix model to optimize the analysis and conduct a systematic construction of the enterprise performance evaluation system. The adoption of new research methods and perspectives to promote the study of the use of performance information is of great significance to achieve the effectiveness, science, and sustainability of corporate performance management. To solve the problem of objectivity and scientificity of performance information use, this part attempts to analyze performance information use from the perspective of the multidimensional big data matrix, focusing on the techniques and methods in the process of promoting performance information use from the multidimensional big data matrix and tries to construct a system model of enterprise performance information use from two dimensions: the use of performance information sources and the use of performance information results. Based on multiple theoretical hypotheses, a theoretical and empirical basis is provided for the division of demand dimensions of enterprise performance evaluation system. Through social capital theory, three dimensions of network social capital, cognitive social capital, and structural social capital are hypothesized, and the logistic regression method is applied for empirical study. The results show that these three dimensions have significant effects on the knowledge demand of enterprise performance evaluation systems. It is verified that the multidimensional big data matrix can enhance the quality of performance information sources and improve the objectivity of performance information. In the performance information source use dimension, the analysis verified that the collection and preprocessing technology of big data can realize the automation, real-time, and diversification of information collection and preprocessing, and enhance the objectivity of performance information. Big data helps to improve the quality and effectiveness of performance information results use. In the dimension of using performance information results, the distributed computing and analysis processing technology of big data can assist the decision support system, and the use of information can be shifted from micromanagement to decision support, to realize the scientific use of performance information and improve the quality of enterprise management decisions.
\end{abstract}

\section{Introduction}

The importance of research on corporate performance management and related topics has received continued academic attention. Corporate performance management, which draws on the methods of business administration, is a new administrative model developed from the Western New Public Management. Since the rise of research, the theory of corporate performance management has developed rapidly, especially in the areas of performance index systems, performance assessment subjects, and performance assess- ment tools. Fruitful research results have been achieved [1]. The academic research results of corporate performance management have been on a growing trend in the last decade or so. Corporate performance management has been one of the important issues in public administration research and a hotspot and key area in the discipline of public management, and public management academics have devoted greater attention and power to research on the topic of corporate performance management [2]. With the popularity of the Internet, artificial intelligence, and 5G technology, big data has entered all occupations. It has not only 
brought great convenience to individuals' lives but also provided new directions for enterprises to think about. Small and medium-sized enterprises are the main body of the structure of the market economy, with a large number, small scale, limited capital, weak risk resistance, and other characteristics [3]. If SMEs want to be invincible in the fierce competition of the market economy, they must adapt to the changes of the times, use big data technology to assist the development of enterprises, and improve their competitiveness. Corporate performance management has always been one of the important issues of public administration research, and it is also a hot and key field of public management disciplines. Public management academic circles have devoted greater attention and strength to the research on corporate performance management topics. As an important part of enterprise management, human resource management has a direct impact on whether the strategic planning of enterprises can be realized. As the core function of $\mathrm{HR}$ management, performance management determines the level of HR management of an enterprise [4]. The development of big data technology brings greater opportunities and challenges to HRM. How to use big data to collect, organize and analyze macro- and microeconomic information to help realize company strategy, promote management level, and make the enterprise invincible in the fierce market competition has become a new issue.

However, the management of enterprises in the context of big data is also facing many new issues. One of the more prominent issues is the performance management of employees and teams. First, the staff and job settings and work processes of the appraisal team in the context of big data are very different from the previous ones. Their main work tasks are real estate data mining, database management, and updating, data platform development and construction, network maintenance, and promotion and aftersales of data products. The difference in work tasks inevitably makes the performance appraisal indexes of employees very different. Traditional performance appraisal indexes focus more on employees' assessment experience and skills, while the use of data assessment systems should focus more on personnel's ability of data analysis and software development. Second, the association between jobs in the context of big data is stronger and requires a higher degree of teamwork. Work tasks include real estate data mining, database management and update, data platform development and construction, network maintenance, and data product promotion and after-sales. Therefore, the traditional performance appraisal system focuses more on the assessment of individual performance, while the performance appraisal of the team should be more important in the context of big data [4]. Again, big data requires the assessment system to have nationwide massive data resources, which inevitably brings about the expansion and development of enterprise organizational structure. The expanding organizational structure brings great difficulty to the performance management of personnel. The headquarters of the enterprise and its subordinate branches as well as the project teams are scattered among many cities in the country, so it is difficult to use a unified performance index assessment system for per- formance management, and the quality of personnel and job positions is very different among different teams. Therefore, the performance appraisal in the context of big data should consider both the correlation between the work of each team and the differences in the economic development of each region [5]. Therefore, the performance appraisal system in the context of big data should be a complex system considering various factors.

To sum up, the traditional performance appraisal system can no longer adapt to the current industry in the context of big data. In the context of big data, the strategic objectives of enterprises have changed a lot, shifting from the original traditional assessment method to the direction of data platform assessment. Therefore, the performance management system of the enterprise should also reflect the strategic objectives of the enterprise. In this way, the value of talents can be better assessed and staff can be motivated to work creatively. Through a scientific and systematic performance management system, managers can understand the overall team's work status and work quality, find problems in time, solve problems, and help managers make correct decisions, which can further improve the company's management level, while a scientific performance appraisal system can also provide a reference basis for staff salary increases and job promotions and help managers discover the company's outstanding talents. The scientific and systematic performance management system can clarify the development direction of the company and the competition between teams, which can greatly improve the work efficiency, and let the employees see that they will get the corresponding reasonable compensation for their hard work, so that they can stimulate their work enthusiasm, continuously improve their workability, and contribute to the further development of the company.

\section{Current Status of Research}

The calculation of weights among various performance indicators has been a difficult problem, and there has always been a lack of uniform calculation standards; therefore, attempts have been made to solve the calculation of weights using the development of corresponding software [6]. On the enterprise side, Intel has proposed a 360-degree performance evaluation appraisal method. Its evaluation dimensions are diversified to ensure a more comprehensive assessment, and it is mainly used to appraise personnel above the middle level. Key performance indicators (KPI) are also widely used at this stage. This method is to divide the goals of the enterprise according to different levels and classes and to find out the key factors or key events, which affect the development of the enterprise, to evaluate the performance level of employees, and through the scientific application of the KPI, the method can effectively improve the management level of the enterprise. The key performance indicator appraisal method is characterized by being relatively simple and easy to operate, so it is more widely used in SMEs [7]. In summary, the balanced scorecard method, the key performance indicator method, the 360degree performance appraisal method, and the goal management method are the commonly used performance appraisal 
methods in foreign companies [8]. These four methods have their advantages and shortcomings, as well as their scope of application. There are more in-depth researches in the theory of performance appraisal management, and some large enterprises have made outstanding explorations in the practice of performance appraisal management [9]. The main problem is that the corporate culture in different regions is different, the assessment methods used, the assessment system established and the assessment indexes also differ, and a unified performance assessment management system has not been established. At present, enterprises often use the key performance indicator method, 360-degree performance appraisal method, etc. The full combination of theory and practice and mutual promotion make this part of the research get continuous and in-depth development [10]. And in recent years, foreign countries have begun to pay attention to performance evaluation research in the organizational context. Researchers in performance appraisal emphasize that performance appraisal is seen as a complex process that includes social context, employee feelings, and cognition. Some experts and scholars have also begun to focus on the study of team performance and have achieved some results. Performance appraisal should consider not only the relevance of the work of each team but also the differences in the economic development of each region. Therefore, the performance appraisal system in the context of big data should be a complex system that considers various factors.

Liu et al. emphasize that firms form a large amount of unique, hard-to-replicate tacit knowledge based on practices, skills, and other resources and transform this knowledge into firm competitiveness through effective accumulation and learning within the organization [11]. Based on this, Zhou et al. argue that dynamic capabilities are processes by which firms use resources, and he points out that dynamic capabilities can not only support firms to adjust their strategies to match the changing environment, but it can even create new markets and improve business performance [12]. In a constantly changing environment, it is difficult to bring lasting benefits to a company with unchanging core competencies. Grant et al. explored the mechanism of corporate knowledge. Xiao et al. argue from the perspective of organizational knowledge evolution that enterprise capability is ultimately the accumulation of empirical knowledge of the enterprise, which is reflected in the business processes of the company's business [13]. The theory of enterprise knowledge is an integration of the resource base theory, core competency theory, and dynamic competency theory. Core competence is a heterogeneous resource of enterprises, while knowledge is the basis of the core competence of enterprises, and the difference of competitive advantages of enterprises originates from the different mechanisms of knowledge creation and application [14]. Enterprises should not only consider enhancing the utility of current knowledge but also seek new knowledge acquisition, and constantly updating knowledge is the key to maintain competitive advantage.

Among the theoretical studies, some scholars mainly explore the realization path of innovative enterprise performance management models utilizing big data thinking and machine learning technologies [15-17]. Perkhofer believes that the characteristics of the era of big data and public sector performance management have a fit and build a simple model of public sector performance management thinking, suggesting scientific use of data mining techniques to build a database system of public sector performance management information [18]. Through the analysis of massive performance data, deeper performance management issues are discovered. It focuses on exploring the influencing factors of applying big data technology [19] in the public sector, proposes a key approach to big data application, presents a theoretical framework to explain the motivation, capability, and performance of big data application in the public sector, and investigates the impact path of big data application on public service performance. It also proposes that future research should construct and test the theoretical framework of the causes, processes, and performance of big data applications in the public sector and make recommendations to promote big data applications in the public sector.

\section{Multidimensional Big Data Matrix Model in Enterprise Performance Evaluation System Optimization Analysis}

3.1. Optimal Design of Multidimensional Big Data Matrix Model. Multidimensional data fusion is an automated information processing approach that brings together multiple sources of information to assist decision-makers in making decisions by transforming the collected information into representational values that can be processed $[20,21]$. The process of data fusion involves many aspects such as data collection, processing, detection, combination, and evaluation. Through data processing, multisource data is fused, so as to accurately identify the state of the event, the safety of the environment, and the participants' identity and other information. The process of data fusion involves numerous aspects of data collection, processing, detection, combination, and evaluation of data from multiple sources to accurately identify information such as the state of an event, the level of safety of the environment, and the identity of the participants. In sorting out the definition of multidimensional data fusion, it can be found that multidimensional data fusion is the process of organizing data from multiple sources, identifying the corresponding knowledge, and removing redundant and useless information, to obtain target state information and rank the importance of things that need to be processed, thus helping users to make decisions. The theory of multidimensional data fusion was first applied to the military field to deal with target tracking and identification, system warning, and situational assessment in military information.

Multidimensional data fusion has also undergone a long period of development and is also characterized as interdisciplinary and cross-disciplinary, a comprehensive discipline involving knowledge and techniques from various fields. Therefore, scholars do not have the same definition of multidimensional data fusion, and the accepted definition for information fusion is that proposed by the Federation of 
Directors of the Tri-Service Organization Laboratory. The fusion of multidimensional data can be performed using Bayesian estimation. When fusing, the data should be as independent as possible. By dividing independently, Bayesian estimation can be used to evaluate the system for decisionmaking. Scholars have different definitions of multidimensional data fusion. For the definition of information fusion, the universally recognized definition is proposed by the United States Tri-Service Organization Laboratory Council.

Suppose that the decisions that can be made in the system are $A 1, A 2, \ldots, A m$, and the observed outcome is denoted as $\mathrm{B}$. This allows the emergent prior knowledge to be used so that the probability of deciding $\mathrm{p}(\mathrm{Ai})$ and the outcome $\mathrm{p}(\mathrm{B} \mid \mathrm{Ai})$ that occurs under a certain decision can be obtained. The probability of an outcome $\mathrm{p}(\mathrm{B} \mid \mathrm{Ai})$ under the probability of a decision can be transformed into a posteriori probability $\mathrm{p}(\mathrm{Ai} \mid \mathrm{B})$ in the Bayesian formulation. This allows the Bayesian formula to be represented using the conditional formula.

$$
p\left(A_{i} \mid B \cup C\right)=\frac{p\left(B \cup C \mid A_{i}\right)}{\sum_{j=1}^{m} p\left(B \cap C \mid A_{i}\right)},
$$

where $B$ is an observation and $C$ is observation under another source. For the decision condition $\mathrm{Ai}$, the probability of occurrence of observation $C, p\left(B \cup C \mid A_{i}\right)$, such that the probability of simultaneous occurrence of $\mathrm{B}$ and $\mathrm{C}$ needs to be calculated. However, this calculation is difficult, so the formula can be further modified. First assume that A, B, and $\mathrm{C}$ are independent of each other.

$$
p\left(B \cup C \mid A_{i}\right)=p\left(B \mid A_{i}\right) p\left(C \mid A_{i}\right) .
$$

Then, the equation is rewritten as equation (3).

$$
p\left(C \mid A_{i} \cup B\right)=\frac{p\left(B \cup C \mid A_{i}\right) p\left(C \mid A_{i}\right)}{\sum_{j=1}^{m} p\left(B \cap C \mid A_{i}\right) p\left(B \mid A_{i}\right)} .
$$

Rough set theory can effectively deal with some noisy data or provide solutions to problems such as incomplete data and inaccurate classification. In the rough set theory, the processing method for knowledge is more comprehensive, and the knowledge granularity can be divided, to ensure the accuracy of knowledge, which is also a knowledge theory domain method. Through rough set theory, it can provide a theoretical basis for the treatment of some uncertainty problems [22]. The coarse and fine-grained knowledge is also the criterion for determining whether knowledge can be accurately divided and whether it can accurately describe the concept of a certain field. Rough set is used as the dividing basis, and knowledge is defined on this basis, to describe the concept of the domain more accurately. The advantage of the rough set theory is that it does not require a priori information, can effectively classify knowledge with known information, and can approximate a large amount of repetitive knowledge, so that it is easier to find law in this knowledge. This feature makes rough set theory widely used in the fields of data mining, expert systems, and pattern recogni- tion, and it can effectively analyze the hidden laws or rules in knowledge without adding any information of data.

By these ideas makes rough sets can be better used in the field of data mining. When performing data mining, it is possible to uncover the fine-grained nature of knowledge and uncover associations between data. Knowledge can be partitioned using rough sets, and this partitioning is often based on a theoretical domain. The coarse and fine granularity of knowledge is also the criterion that determines whether knowledge can be accurately classified and whether it can accurately describe the concepts of a domain. Using rough sets as the basis for division, knowledge is defined on this basis to describe the concepts of the domain more accurately, as shown in Figure 1.

Cross-source multidimensional government data sharing is an extension of the traditional concept of government data, and its data-sharing model architecture is also significantly different from the traditional model architecture, mainly including three extensions: the data of business systems are different, including the data of many departments such as public prosecution, law enforcement, government affairs, and industry and commerce administration. When constructing the system, some of the data come from the self-built systems of the units at this level, while some originate from the national and provincial vertical systems, in addition to the interface between the self-built system and the data sharing and exchange platform, the technology of data pipeline is also used to realize the interface between the national vertical system and the data-sharing platform. Part of the data comes from the self-built system of the unit at the same level, and part comes from the national and provincial vertical systems. Therefore, when building the system, in addition to the connection between the self-built system and the data sharing and exchange platform, the data pipeline technology is used to realize the national vertical system, interfacing with the data-sharing platform. The diversity of data structure includes structured, semistructured, and unstructured data, such as structured data of business processing in the governmental system, semistructured data of system logs, and unstructured data of each governmental monitoring system.

$$
B E L(A)=\sum m(B)^{2}
$$

The virtual knowledge community contains a large amount of user behavior data and personalized demand data. These data constitute the multidimensional data of the virtual knowledge community. Using these data for fusion can alleviate the data sparsity and cold-start problems in traditional recommendation systems, and therefore, hybrid recommendation with fused multidimensional data is an important means for a virtual knowledge community to solve user information overload. When dealing with multidimensional data, it often faces situations such as missing data or unknown data, so it is necessary to predict the filling of missing data or unknown data based on known data. Clustering methods can be used to cluster the data and predict the relationship between missing data using existing 


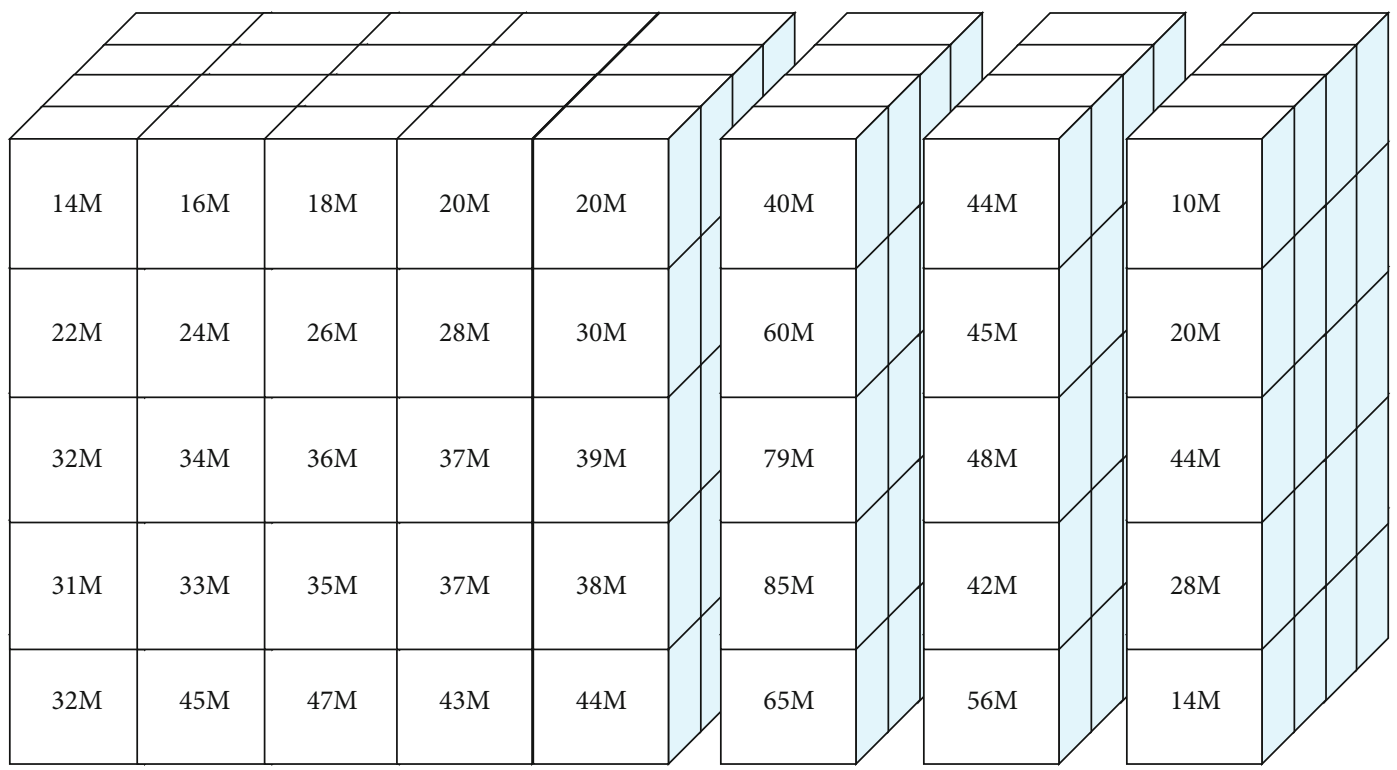

Figure 1: Multidimensional big data matrix model.

relationships, thus considering the correlation between data, and the process of calculation is easier to understand. Missing data can also be filled using a method based on the expected maximum. You can also use methods based on the expected maximum to fill in missing data. This is a feature that uses nonnormal distribution and uses a kernel function to calculate the data distribution density. This is a feature that uses nonnormal distributions and calculates the density of the data distribution using kernel functions. The classifier in Bayes and the expected maximum can predict the data to be filled. There are also studies on missing data filling methods based on self-associating neural networks, which can obtain data with higher accuracy.

The cross-source multidimensional government datasharing influence factors are $n$, and each factor is evaluated by $m$ levels, then the set of influence factors to be classified. Where each $u$ is a set of factor evaluation vectors, representing the set of influence degree measures of the $k$ th factor. The study object matrix can be obtained, as shown in equation (5).

$$
X_{n \times m}=\left[\begin{array}{cccc}
x_{11} & x_{12} & \cdots & x_{1 m} \\
x_{21} & x_{22} & \cdots & x_{2 m} \\
\cdots & \cdots & \cdots & \cdots \\
x_{n 1} & x_{n 2} & \cdots & x_{n m}
\end{array}\right] .
$$

To reduce the problem of "swallowing small numbers by large numbers" when comparing data of different orders of magnitude, the original data need to be normalized to the interval $[0,1]$ by data normalization. The $j$ th column of the matrix $X$ is normalized, see (6).

$$
x_{i j}^{\prime}=\frac{x_{i j}+\max \left\{\mathrm{x}_{i j}\right\}}{\max \left\{\mathrm{x}_{i j}\right\}+\min \left\{\mathrm{x}_{i j}\right\}} .
$$

The reference series and the evaluation object are standardized by the data and the correlation coefficient of the corresponding index between the j-rated evaluation index of the $i$ th factor and the reference series in equation (7).

$$
\xi_{i j}=\frac{\max \min \left\{\mathrm{x}_{i j}\right\}}{\max \left\{\mathrm{x}_{i j}\right\}+\min \left\{\mathrm{x}_{i j}\right\}} .
$$

To calculate the correlation degree, the influence degree of different indicators in the research object needs to be considered comprehensively, so the indicator weights are introduced, as shown in Figure 2, which is the weight of the influence level of each influence factor, and the correlation coefficient of all indicators of each factor can be combined into one correlation degree, and the correlation degree can be obtained after considering the weights.

$$
r_{i}=\sum_{j=1}^{m} p_{j} \xi_{i}(j)
$$

Performance information is characterized by the properties of information itself but also has some special characteristics of performance management. The characteristics of performance information include purpose, accuracy, timeliness, formality, consistency, and completeness. The purpose of performance information is that performance information is not blind and random information but has a specific purpose. Accuracy of performance information means that it can accurately fit the purpose of performance management and meet the needs of performance management. The earlier performance information is obtained, the greater the effect; conversely, the later the time, the weaker the function of the information. The formality of performance information is the value of performance information to realize evaluation and judgment. Timeliness can also be understood as timeliness. Performance information reflects the status of 


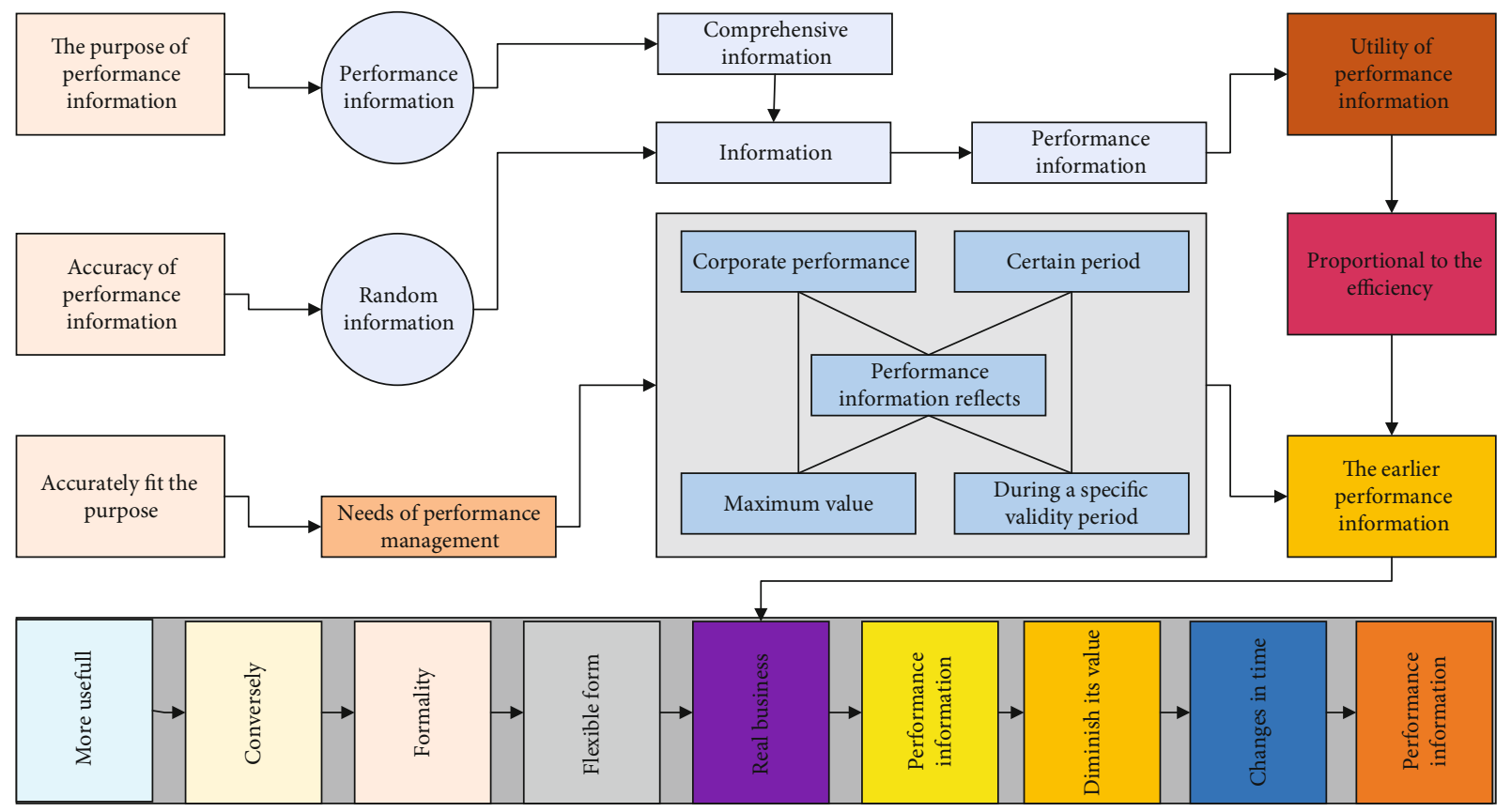

FIGURE 2: Design framework.

corporate performance within a certain period. It has maximum value only during a specific validity period. Therefore, its value is closely related to time, and the utility of performance information is directly proportional to the efficiency of its supply. The earlier performance information is obtained, the more useful it is; conversely, the later it is obtained, the less useful it will be. The formality of performance information is that to achieve the value of evaluation and judgment, performance information needs to be in a clear and flexible form to show the real business management situation in all aspects. Consistency is that performance information does not diminish its value because of changes in time and place. Of course, more importantly, performance information must be complete. The performance of business management requires complete and comprehensive information, so the information cannot be left out; otherwise, it will bring a negative impact on the use of performance information results.

\subsection{Experimental Design of Enterprise Performance} Evaluation System. The goal of performance management based on big data technology is to improve the accuracy and credibility of performance management using big data technology, make counseling more targeted through timely feedback, and ultimately achieve the purpose of mobilizing employees' work motivation and creativity, improving individual performance, and enhancing the overall performance level of the enterprise. Unlike the traditional performance management model, the data source channels of HR performance management based on big data technology are diverse [23]. The source of data information no longer relies on the original data only by manual entry but directly extracts the performance management information of a certain time point automatically through the system. The form of data information is richer and more diversified, covering information such as graphics, reports, figures, tables, and sounds in addition to conventional text carriers, which expand the dimensionality of data sources and are a fusion of structured data and unstructured data and cannot be concluded using statistical analysis only, so it is necessary to organize, analyze, and store this information through big data technology to complete the informatization process. To be used by managers in general big data is stored on an open cloud platform, which provides a data source for collecting data and sharing data and provides support for the openness of performance management data. The openness is reflected from two aspects: on the one hand, it is open to the users of performance data, providing help to those who need to use the data for decision analysis within the enterprise, reducing the cost of acquiring data for the enterprise, and helping to improve the accuracy of their decisions; on the other hand, it is open to the uploaders, enabling the platform to continuously collect more data and cover a wider range of data, as shown in Figure 3.

The sharing of performance management data can realize data transparency and reduce the cost of acquisition that occurs due to information asymmetry. Data information reaches employees and managers directly, and employees can check their performance results at any time and learn about the performance results of others, so that employees can know themselves and their opponents, which can play the role of self-checking and supervision, and find the gap with others in time and motivate each other to learn and improve work motivation. Performance management based on big data can realize real-time performance feedback and automation. As shown in Figure 3, the performance management based on big data technology can chase the treadle on aj method in real-time when the fruit exceeds the preset 


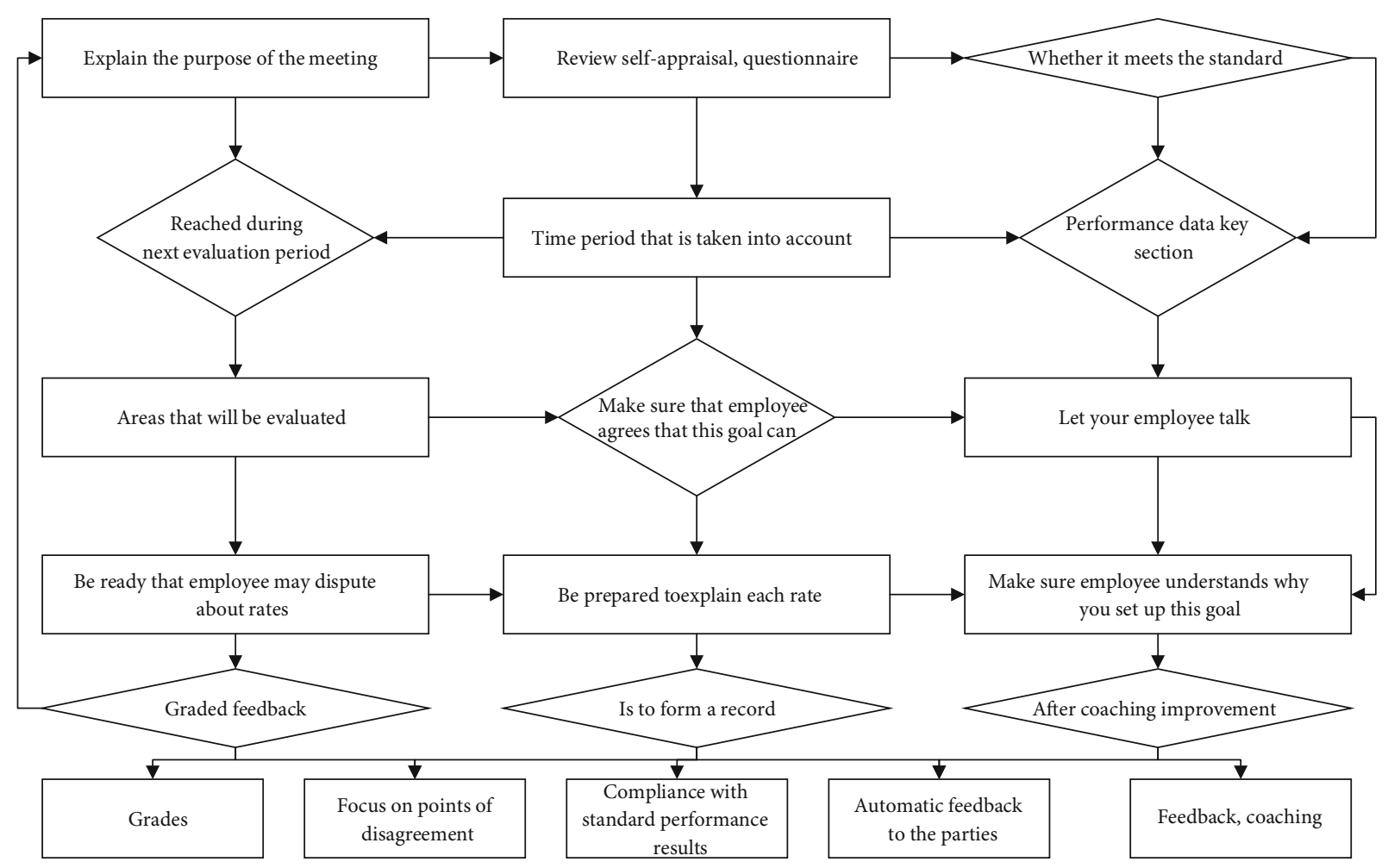

Figure 3: Performance feedback process.

value; the system will automatically issue the results for comparison and analysis through human-computer interaction $[24,25]$. If the assessment results do not reach the precorrected instructions, the system will send the results to the person concerned for guidance and feedback; if the assessment results do not reach the preset indicators, the system will send the results to the supervisor at the same time, so that the supervisor can understand the current situation of employees' work and conduct targeted counseling on time [26].

For the performance appraisal and feedback process, all data are made by database for data custody, collecting more complete information and more accurate appraisal results. The performance appraisal management system based on big data can effectively help employees conduct self-examination, correct bias, and improve work efficiency. Performance management based on big data technology realizes real-time tracking, data collection, comparison, analysis, and feedback so that the level of human resources performance management is greatly improved.

At present, many local enterprises still lack a scientific reward and punishment system, and the incentive validity of performance information use is not high. In terms of incentive direction, there are more positive incentives than negative incentives in the use of performance information for corporate civil servants. In many cases, the results of performance evaluation are generally satisfactory. Among the incentive approaches, the positive incentive of job promotion is significantly more than the other incentive approaches, which has a stimulating effect on civil servants' officialism. Data information forms are more abundant and diversified. In addition to conventional text carriers, it also covers graphics, reports, numbers, tables, sounds, and other information. These data expand the dimensions of data sources and are the fusion of structured data and unstructured data. The unbalanced and unscientific setting of incentive methods is often unable to properly guide the attitudes and behaviors of civil servants.

Insufficient degree of disclosure of performance information results to subjects external to the enterprise. At present, the content and scope of performance information disclosed to the public in practice are still inadequate and insufficient. The performance information is mainly transmitted and shared within the enterprise organization, and the relatively high degree of public disclosure is mainly for online evaluation and enterprise satisfaction information, but the degree of disclosure of other information related to people's livelihood is relatively low, as shown in Figure 4.

The selected expert should be an authority in the field of corporate performance evaluation research or an expert scholar with relevant work experience to ensure the accuracy of the evaluation results. In addition, the selected experts should have relevant research or understanding of the new retail innovation ecosystem. The experts should preferably have experience in researching innovation ecosystem projects, and experienced new retail workers should preferably be financial directors or regional directors. The number of experts should be neither too many nor too few. If the number of experts is too large, on the one hand, the cost is relatively high and the feedback period will be extended accordingly; on the other hand, if the opinions of experts are too diverse in the comprehensive processing, not only 


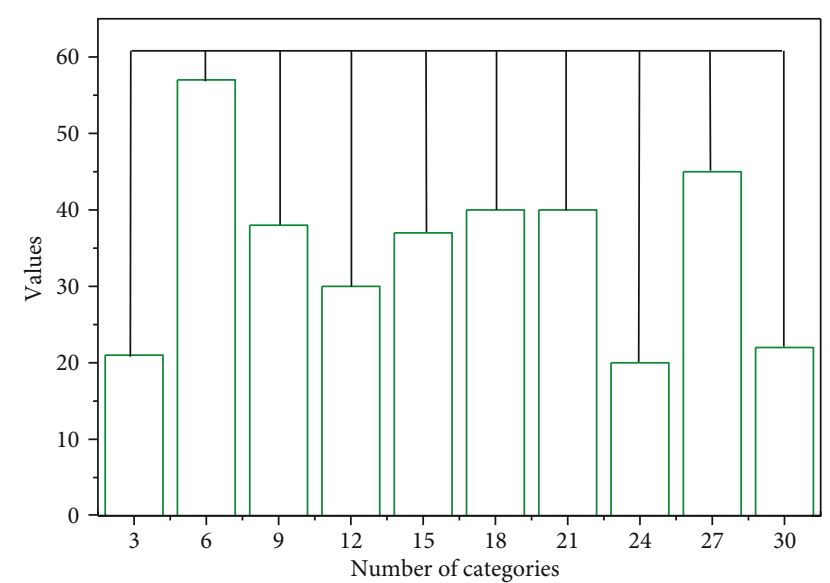

FIGURE 4: Dynamic clustering diagram of factors influencing crosssource multidimensional government data sharing.

is it time-consuming and labor-intensive, but it may not even be possible to reach a unified conclusion. If the number of experts is too small, the analysis results will not only be insufficient for scientific accuracy, but the whole study will be meaningless. Some studies have shown that the number of experts should be best at 7 . When the number of experts exceeds 7 , the accuracy of the evaluation results does not improve significantly with the number of people, so it is better to choose 7 experts.

\section{Analysis of Results}

4.1. Results of the Optimized Multidimensional Big Data Matrix Model. The measurement method of the government recommendation system contains three aspects; one is the comparison of the calculation results of multiple recommendation models. The other is the scoring prediction for result measurement. The recommendation results of a certain enterprise are extracted for manual verification. This study compares the results of enterprise-based recommendation, matter-based recommendation, SVD-based recommendation, and SVD++-based recommendation, with $90 \%$ of the original data as training data and $10 \%$ of the randomly selected data, as test data, as shown in Figure 5.

By comparing several different algorithms to validate G2B recommendations based on cross-source multidimensional government data, it is appropriate to use the SVD++ algorithm combining enterprise recommendations and matter recommendations as to the core recommendation algorithm. The higher recall rate and accuracy rate in the algorithm comparison indicate that the algorithm is more effective and the lower differential mean evaluation is better. $90 \%$ of the original data is used as training data, and $10 \%$ of the data is randomly selected. From Figure 5, it can be found that the recall rate and the check accuracy rate are much higher for both the firm-based recommendation and the matter-based recommendation compared to someone, KNN, SVD, and SVD++. Due to the sparsity of the matrix, the enterprise-based recommendation and matter based recommendation have the situation that some enterprises can- not calculate the results, while SVD++ achieves all enterprise recommendations by dimensionality reduction but its results are less accurate, so these three methods are combined to achieve the complementary and optimized results. Combining enterprise recommendation, matter recommendation, and SVD++ recommendation is a kind of hybrid recommendation combining multiple algorithms, which is to merge and optimize the ranking of multiple calculation results. The algorithm used in this paper is reasonable and effective by comparing the three dimensions of recall rate, check accuracy rate, and differential mean evaluation.

To be able to clearly describe the input-output efficiency of each sector of the enterprise, this study takes each sector of the enterprise as a decision unit in 2020, constructs the CCR model, BCC model, and super-efficiency model, and uses MATLAB software for calculation. By calculating the comprehensive efficiency, pure technical efficiency, scale efficiency, and super efficiency values, the comparative analysis of the input-output efficiency of each enterprise sector in 2020 is carried out, and the calculation results are shown in Figure 6.

The lower the efficiency value, the more serious the waste of resources. In particular, the overall efficiency of the Agriculture Bureau and the Environmental Protection Bureau is lower than 0.7 , which is relatively low. The comprehensive efficiency in Figure 6 is calculated by the CCR model, and the results show that the comprehensive efficiency values of Education Bureau, Quality and Technical Supervision Bureau, Public Security Bureau, and Health and Family Planning Bureau are all equal to 1 , which means that the DEA of these departments is relatively effective, indicating that these four departments do not have pure technical inefficiency or scale inefficiency, indicating that these departments have achieved effectiveness in terms of technology and scale. This indicates that these sectors are effective in terms of technology and scale and that they can achieve efficient and solid returns to scale with proper inputs in the process of operation. In addition to these four departments, the other three departments: Administration for Industry and Commerce, Bureau of Agriculture, and Environmental Protection Bureau have integrated efficiency values less than 1. They all have different degrees of integrated efficiency inefficiency, indicating that these departments have some degree of resource waste, and some of the input resources cannot be effectively transformed into outputs, and some of the resources invested in the data sharing work do not play the proper value. In particular, the comprehensive efficiency of the Agriculture Bureau and the Environmental Protection Bureau is below 0.7, which is low in relative terms. Low overall efficiency may be caused by managerial errors and unreasonable scale, and technical efficiency can be improved by optimizing resource allocation and improving resource management methods.

According to the calculation of the BCC model, the scale efficiency values of the Bureau of Quality and Technical Supervision, the Bureau of Education, and the Bureau of Health and Family Planning are all lower than reasonable levels, which indicates that there is a waste of resources in the construction process, so it is recommended that these 


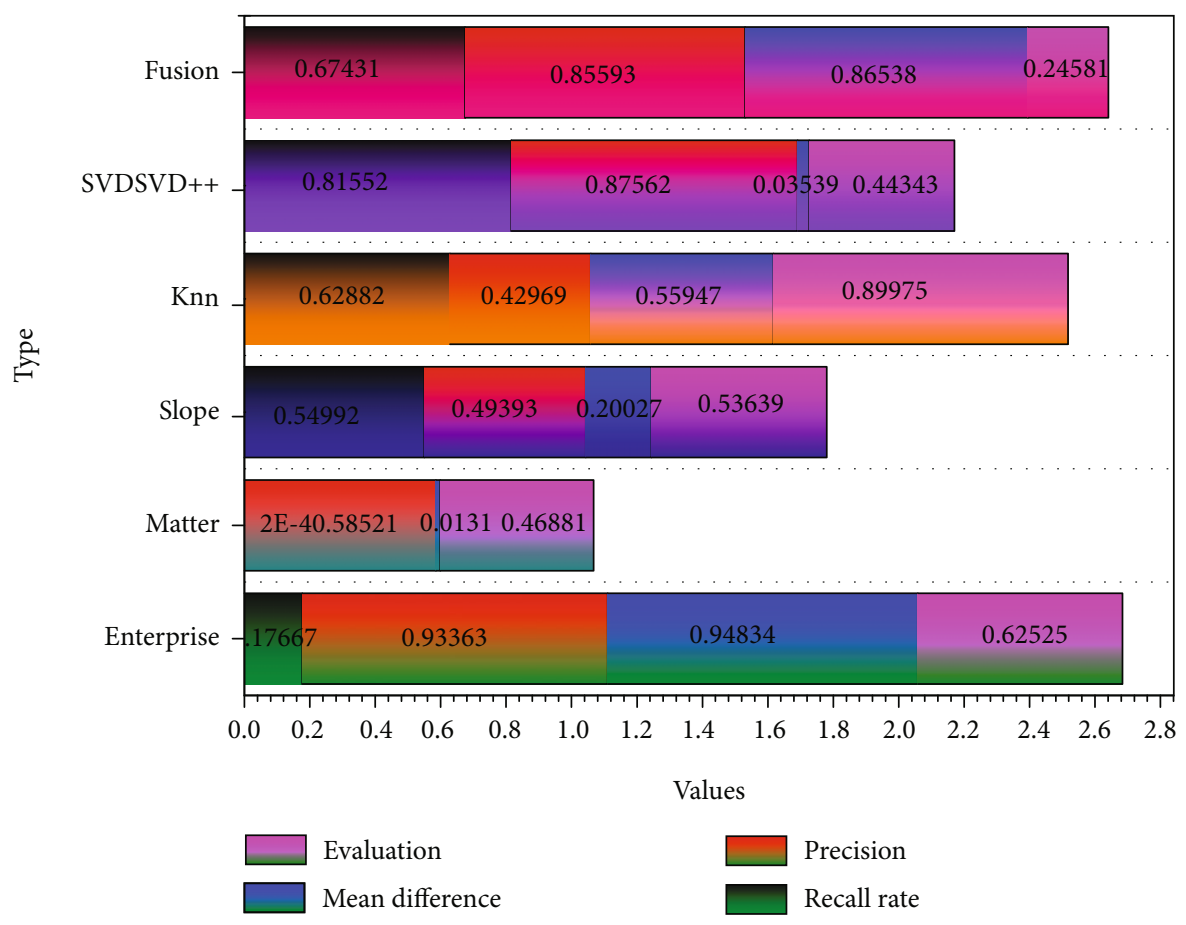

FIgURE 5: Comparison of the efficiency of various algorithms.

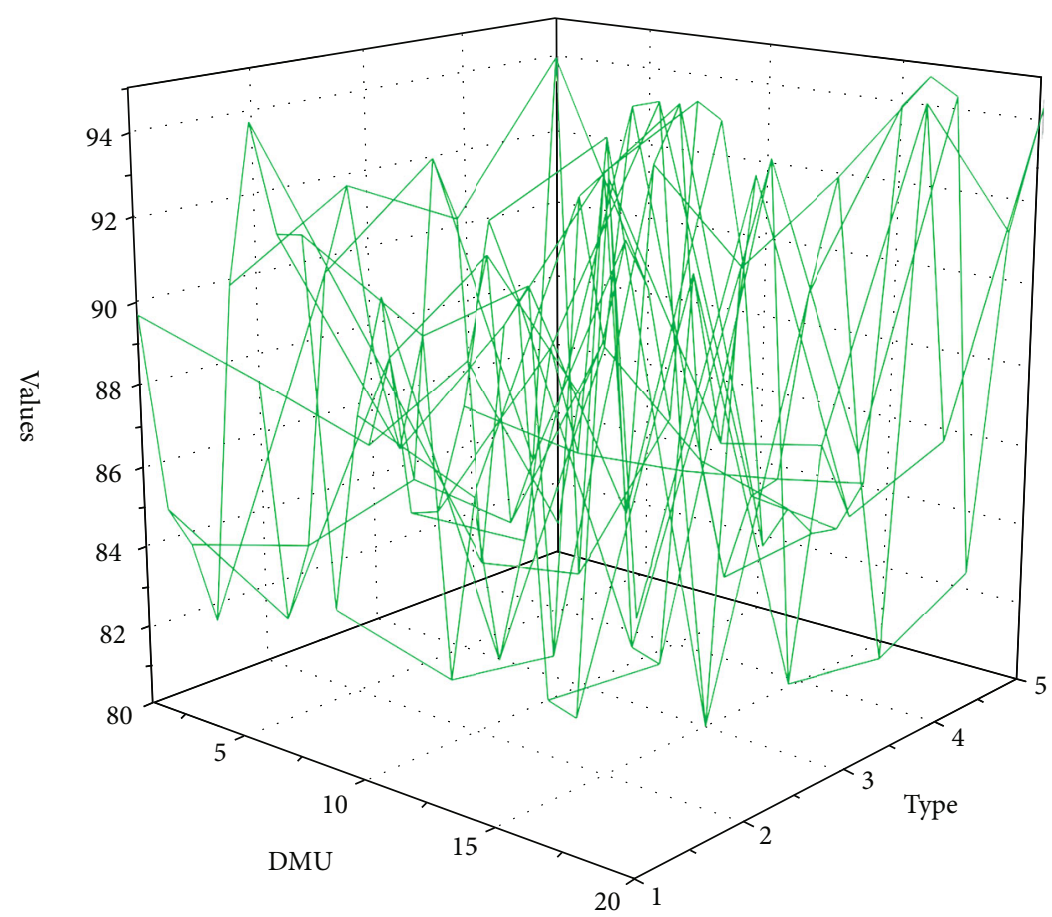

FIGURE 6: Efficiency evaluation value of each department.

units do a good job of managing the whole process in the cross-source multidimensional data sharing work. This includes top-level planning at the early stage of the project, consulting and design at the system implementation stage, and more comprehensive and careful management of the bidding process. We should hire professional consulting companies, supervisory companies, bidding companies, and software evaluation companies to do a good job and conduct forward-looking research jointly with universities and professional research institutions to promote the sharing of cross-source multidimensional data and actively play the value of data application. 

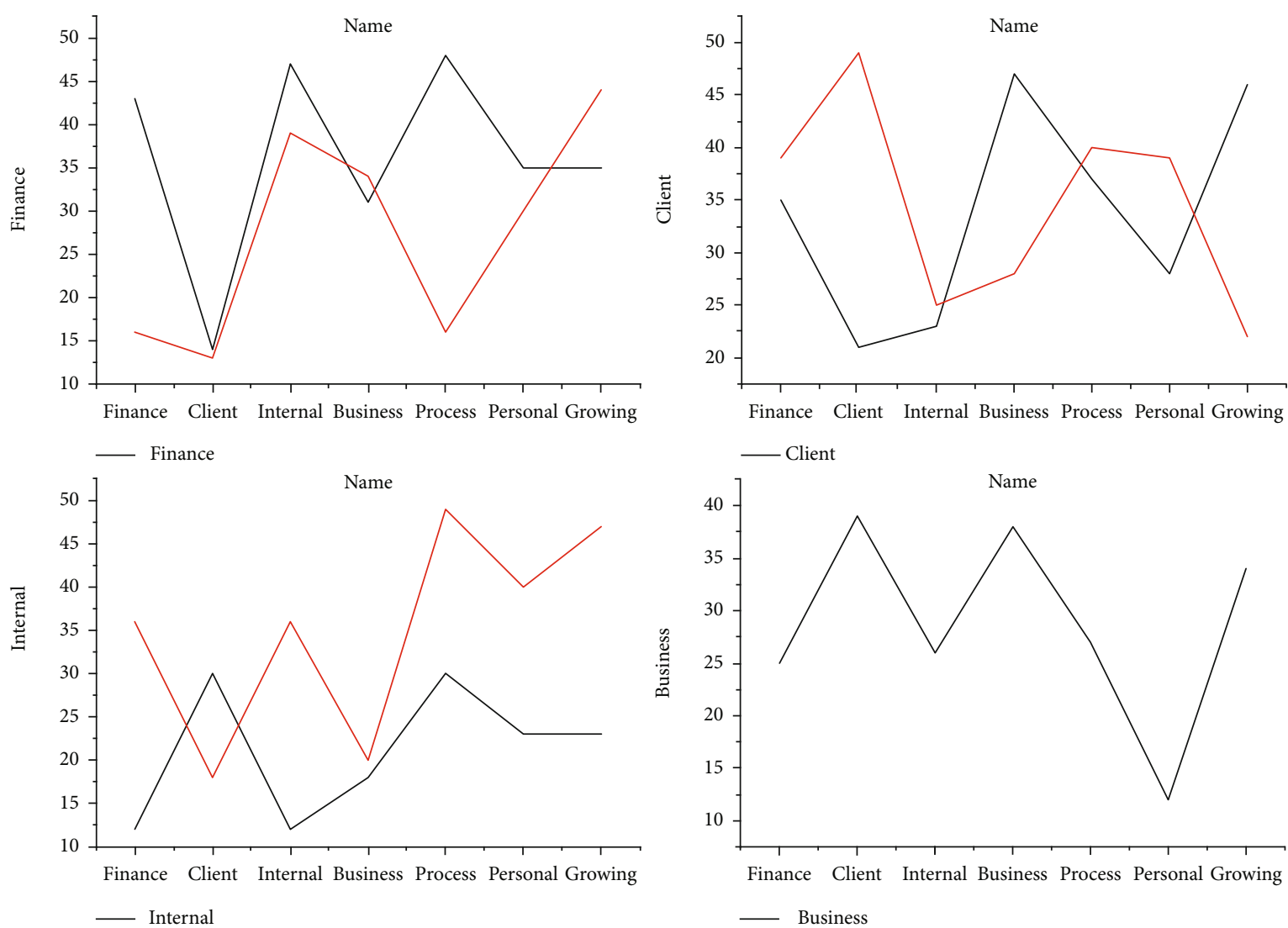

Figure 7: Judgment of the weighting factor of the first-level index.

4.2. Experimental Results. A two-dimensional table consisting of rows and columns is constructed for the performance indicators of the same level to be evaluated, and all the performance indicators to be evaluated at the same level are listed in the rows and columns, respectively. The following is an example of the construction method of the weight factor judgment table for the first level performance indicators of the data team performance evaluation system. As shown in Figure 7, the first column lists the four levels 1 performance indicators of the data team, which are financial level, customer level, internal business process level, and personal growth level. The first column also lists these four indicators.

Each cell at the intersection of the rows and columns is filled with a score that compares the importance of the row and column indicators to each other. The criteria and range of comparative scores were filled in by an expert panel of 18 experts based on the quantitative scale of importance. For example, if an expert think that the customer-level indicators are less important than the financial-level indicators, he/she fills in the cell where the customer-level and financial-level indicators are intersected with the value 4 . When all the tables are filled, the table of the weighting factors of the first-level indicators of an expert is formed. The table of 18 levels 1 index weighting factors is formed. The arithmetic mean of the values in the 18 cells of the table of the weighting factors of the first-level indicators is calculated, and the final table of the weighting factors of the first-level indicators of the data team is formed. It is impor- tant to note that the 18 experts' scores need to be checked for consistency, and if they pass, the next step can be performed. Similarly, for the four dimensions of the balanced scorecard, all the following second-level indicators are also used in the same way to construct the weighting factor judgment table separately, and the final weighting factor judgment will be formed.

It is also necessary to combine the weights of the indicators of the two tiers, i.e., the indicator weights of the second tier are multiplied with the corresponding weights of the first tier, which is called the total arrangement of weights. The specific calculation method is to multiply the weights of the second tier ( $\mathrm{C}$ tier) by the respective corresponding weights of the first tier (B tier) to obtain the final team performance index weights. The team performance appraisal results can be analyzed to adjust the pay level of the team. According to the different results of team performance appraisal, it can be divided into the adjustment of overall team salary level and the adjustment of salary level of some positions. The adjustment of the overall salary level of the team is made according to the comprehensive score of the team performance appraisal results. And the adjustment of the salary level of some positions is based on the completion of each performance appraisal index. The principle of salary level adjustment is to first determine the overall performance level of the team according to the total score of the team's performance appraisal results and, then, adjust the salary of some positions according to the completion of each 


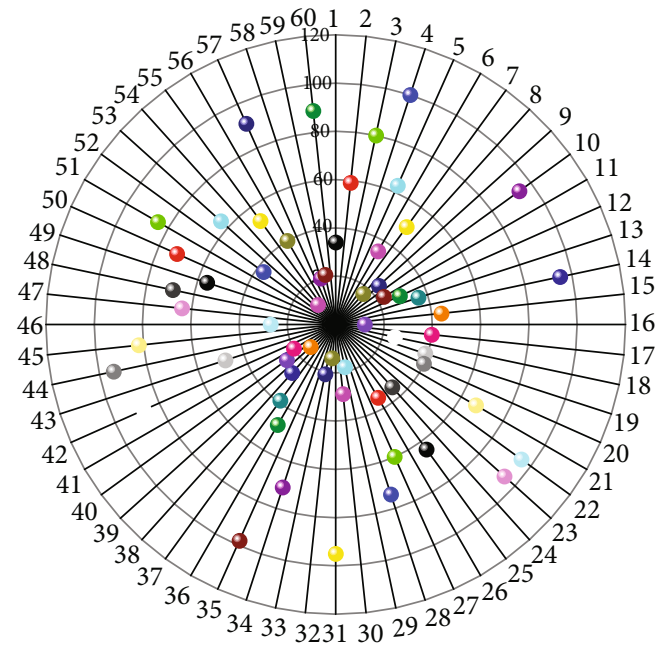

Figure 8: Radar chart of performance elements at the same level.

performance appraisal index. The principle of salary level adjustment is to first determine the overall team performance level based on the total score of the team performance appraisal results and, then, to adjust the salary of some positions according to the completion of each performance appraisal index.

The evaluation of $R \& D$ personnel should focus on the combination of result-oriented quantitative and qualitative indicators, and the quantitative evaluation should be result-oriented, considering the R\&D process, while focusing on the realization of $\mathrm{R} \& \mathrm{D}$ personnel's self-worth, so that the performance of $\mathrm{R} \& \mathrm{D}$ personnel can be truly and reasonably evaluated. For the qualitative part, I can ask experts and relevant personnel to depict a radar chart of performance evaluation elements for R\&D personnel's job competency and then conduct a comprehensive analysis by combining big data technology to combine the qualitative part with the quantitative output part, to determine the most reasonable index weights. The radar diagram of $\mathrm{R} \& \mathrm{D}$ personnel performance evaluation is shown in Figure 8.

Risk early warning is a system that monitors changes in the trend of risk factors based on the characteristics of the object of study, collects related information, and measures the strength of deviation from the warning line for each type of risk state and, at the same, time adopts preemptive measures for decision-makers and emits early warning signals. The construction of a risk warning system requires the establishment of an indicator measurement system and indepth analysis and processing of indicators. The evaluation indicator system in HR performance management is based on performance evaluation indicators and risk indicator evaluation levels, which are defined digitally, and the early warning system indicators are included in the performance indicator design considerations, which is the basis for the establishment of the early warning system.

Second, according to the actual situation of the enterprise, combined with the performance evaluation index, use reasonable early warning data processing method, set early warning processing model, and comprehensive evalua- tion data index system. The risk model can be set according to the position, work style, and process, generally; there are position risk model, work risk model, process risk model, other models, etc. Finally, according to the degree of completion of performance indicators in the actual work and early warning system indicators to compare, set the early warning interval, when the gap between the actual completion of indicators and early warning indicators exceeds the early warning interval, the early warning system will measure the risk to formulate processing countermeasures and send to the executive and supervisory personnel for the relevant personnel to correct the deviation.

\section{Conclusion}

The positive impact of big data fusion capability on enterprise innovation performance has not been verified. To explore the reason, we can return to the definition of "big data convergence capability", which is the orderly integration of specific business and big data systems, which will break the traditional management style, redeploy and optimize enterprise resources, enhance business process sharing and interactivity, and thus improve the efficiency of enterprise operation. Realizing the orderly combination of business and big data systems, as well as the redeployment and optimization of enterprise resources is a gradual process, which cannot be completed in a short time. At present, China's big data industry is in the primary development stage, especially the relevant technology is in the stage of technical barriers to overcome, and the external conditions for cultivating the enterprise's big data integration capability have not reached a mature time. Therefore, the positive impact of big data integration capability on the innovation performance of enterprises has not been revealed yet. Big data strategic collaboration capability is a positive adjustment of enterprises' strategies in the new era of change. In the fierce market competition, big data strategic collaboration capability enhances the adaptability of enterprises to the market environment, and the actual needs of customers support the concrete implementation of enterprise strategies based on the guidance of learning mechanisms and promote the management innovation activities of enterprise management members from the perspective of internal management and operation. Big data strategic collaboration capability is an important path for the transformation of enterprise innovation performance, providing support for the future development strategy of the enterprise and realizing the improvement of the overall competitiveness of the enterprise.

\section{Data Availability}

The data used to support the findings of this study are included within the article.

\section{Conflicts of Interest}

The author does not have any possible conflicts of interest. 


\section{References}

[1] Z. H. Munim, M. Dushenko, V. J. Jimenez, M. H. Shakil, and M. Imset, "Big data and artificial intelligence in the maritime industry: a bibliometric review and future research directions," Maritime Policy \& Management, vol. 47, no. 5, pp. 577-597, 2020.

[2] J. Wen, J. Yang, B. Jiang, H. Song, and H. Wang, "Big data driven marine environment information forecasting: a time series prediction network," IEEE Transactions on Fuzzy Systems, vol. 29, no. 1, pp. 4-18, 2020.

[3] H. Patel, D. Prajapati, D. Mahida, and M. Shah, "Transforming petroleum downstream sector through big data: a holistic review," Journal of Petroleum Exploration and Production Technology, vol. 10, no. 6, pp. 2601-2611, 2020.

[4] X. Zhu and Y. Yang, "Big data analytics for improving financial performance and sustainability," Journal of Systems Science and Information, vol. 9, no. 2, pp. 175-191, 2021.

[5] V. Kumar and D. Ramachandran, “Developing firms' growth approaches as a multidimensional decision to enhance key stakeholders' wellbeing," International Journal of Research in Marketing, vol. 38, no. 2, pp. 402-424, 2021.

[6] S. A. Gawankar, A. Gunasekaran, and S. Kamble, "A study on investments in the big data-driven supply chain, performance measures and organisational performance in Indian retail 4.0 context," International Journal of Production Research, vol. 58, no. 5, pp. 1574-1593, 2020.

[7] S. Thudumu, P. Branch, J. Jin, and J. Singh, "A comprehensive survey of anomaly detection techniques for high dimensional big data," Journal of Big Data, vol. 7, no. 1, 2020.

[8] S. Chehbi-Gamoura, R. Derrouiche, D. Damand, and M. Barth, "Insights from big Data Analytics in supply chain management: an all-inclusive literature review using the SCOR model," Production Planning \& Control, vol. 31, no. 5, pp. 355-382, 2020.

[9] F. Mohammadi and M. Sahraei-Ardakani, "Multidimensional scenario selection for power systems with stochastic failures," IEEE Transactions on Power Systems, vol. 35, no. 6, pp. 4528-4538, 2020.

[10] L. Ismail and H. Materwala, "Computing server power modeling in a data center," ACM Computing Surveys (CSUR), vol. 53, no. 3, pp. 1-34, 2020.

[11] Z. Liu, N. Wu, Y. Qiao, and Z. Li, "Performance evaluation of public bus transportation by using DEA models and Shannon's entropy: an example from a company in a large city of China," IEEE/CAA Journal of Automatica Sinica, vol. 8, no. 4, pp. 779-795, 2020.

[12] X. Zhou, W. Liang, K. I.-K. Wang, and L. T. Yang, "Deep correlation mining based on hierarchical hybrid networks for heterogeneous big data recommendations," IEEE Transactions on Computational Social Systems, vol. 8, no. 1, pp. 171-178, 2020.

[13] Q. Xiao, M. Shan, M. Gao, X. Xiao, and H. Guo, "Evaluation of the coordination between China's technology and economy using a grey multivariate coupling model," Technological and Economic Development of Economy, vol. 27, no. 1, pp. 24-44, 2021.

[14] W. Höpken, T. Eberle, M. Fuchs, and M. Lexhagen, "Improving tourist arrival prediction: a big data and artificial neural network approach," Journal of Travel Research, vol. 60, no. 5, pp. 998-1017, 2021.
[15] J. Zhang, Y. Liu, H. Liu, and J. Wang, "Learning local-global multiple correlation filters for robust visual tracking with Kalman filter redetection," Sensors, vol. 21, no. 4, p. 1129, 2021.

[16] Y. Jiang, X. Gu, D. Wu, W. Hang, J. Xue, and S. Qiu, “A novel negative-transfer-resistant fuzzy clustering model with a shared cross-domain transfer latent space and its application to brain CT image segmentation," IEEE/ACM Transactions on Computational Biology and Bioinformatics, vol. 18, no. 1, pp. 40-52, 2021.

[17] M. Gao, W. Cai, and R. Liu, "AGTH-net: attention-based graph convolution-guided third-order hourglass network for sports video classification," Journal of Healthcare Engineering, vol. 2021, 2021.

[18] L. Perkhofer, W. Conny, and P. Hofer, "Does design matter when visualizing Big Data? An empirical study to investigate the effect of visualization type and interaction use," Journal of Management Control, vol. 31, no. 1-2, pp. 55-95, 2020.

[19] Z. Wang, P. Zhang, W. Sun, and D. Li, "Application of data dimension reduction method in high-dimensional data based on single-cell 3D genomic contact data," ASP Transactions on Computers, vol. 1, no. 2, 2021.

[20] C. Zhao, L. Ren, Z. Zhang, and Z. Meng, "Master data management for manufacturing big data: a method of evaluation for data network," World Wide Web, vol. 23, no. 2, pp. 14071421, 2020.

[21] W. Cai, Y. Song, and Z. Wei, "Multimodal data guided spatial feature fusion and grouping strategy for E-commerce commodity demand forecasting," Mobile Information Systems, vol. 2021, 2021.

[22] H. Li, X. Bu, X. Liu et al., "Evaluation and prediction of blast furnace status based on big data platform of ironmaking and data mining," ISIJ International, vol. 61, no. 1, pp. 108-118, 2021.

[23] A. Li and R. C. Bergan, "Clinical trial design: past, present, and future in the context of big data and precision medicine," Cancer, vol. 126, no. 22, pp. 4838-4846, 2020.

[24] C. Yan, G. Pang, X. Bai et al., "Beyond triplet loss: person reidentification with fine-grained difference-aware pairwise loss," IEEE Transactions on Multimedia., p. 1, 2021.

[25] W. Sun, P. Zhang, Z. Wang, and D. Li, "Prediction of cardiovascular diseases based on machine learning," ASP Transactions on Internet of Things, vol. 1, no. 1, pp. 30-35, 2021.

[26] Q. G. Shao, J. J. H. Liou, S. S. Weng, and P. Su, "Constructing an entrepreneurship project evaluation system using a hybrid model," Journal of Business Economics and Management, vol. 21, no. 5, pp. 1329-1349, 2020. 